\title{
Dependence of landmine radar signature on aspect angle
}

ISSN 1751-8784

Received on 30th September 2016 Revised 21st December 2016 Accepted on 23rd January 2017 E-First on 13th April 2017 doi: 10.1049/iet-rsn.2016.0491 www.ietdl.org

\author{
Federico Lombardi ${ }^{1}$, Hugh D. Griffiths ${ }^{1}$, Lauren Wright ${ }^{2}$, Alessio Balleri ${ }^{3}$ \\ ${ }^{1}$ Department of Electronic \& Electrical Engineering, University College London, London WC1E 6BT, UK \\ ${ }^{2}$ Australian Defence Organisation, 1004/80 Clarendon Street, Southbank, VIC, AUS 3006, Australia \\ ${ }^{3}$ Centre for Electronic Warfare, Information and Cyber, Cranfield University, Defence Academy of the UK, Shrivenham SN6 8LA, UK \\ «E-mail:f.lombardi@ucl.ac.uk
}

\begin{abstract}
Antipersonnel landmines have been indiscriminately used since World War II, and their long-term persistence in the ground creates a barrier to development in a large number of countries and forces people to live in constant fear. There is a growing demand for reliable landmine detection and localisation systems to return affected areas to their normal use. Due to its ability of detecting both metallic and non-metallic objects, ground penetrating radar (GPR) is a meaningful method for detecting landmines that may allow faster and safer operations. Unlike common clutter objects, most landmines can be modelled as multiple layered dielectric cylinders that cause multiple interfering reflections and result in features with a characteristic angular pattern. Due to this, landmines are expected to produce signatures that present some discriminant features that could be used for reducing the GPR false alarm rate. In this study, measurements of three inert landmines have been carried out to study and characterise landmine signatures as a function of polarisation angle and aspect angle.
\end{abstract}

\section{Introduction}

Landmine contamination is one of the most unacceptable threats posed to humanity [1]. Landmines have been spread in an uncontrolled way in many zones of conflict and they pose a significant humanitarian risk for civilians, and in particular children and refugees $[2,3]$. In addition to landmines, unexploded abandoned ordnance, cluster bombs, submunitions and improvised devices also remain active after the end of a conflict. These are left unstable, highly explosive, and sometimes contain incendiary materials that can create long-term damage to affected lands and communities [4]. Post-conflict recovery of areas affected by landmines can only start once explosive remnants of war and landmines have been located and removed and this poses an important problem to the economic development of the affected populations [5].

The primary goal of demining operations is to safely return an affected area to its normal use. One of the problems with demining is that technology, to date, has had only a marginal impact on mine action equipment, in particular for humanitarian operations [6]. Demining operations have been largely conducted by using manual probes, sniffer dogs and metal detectors. The use of a large variety of sensors has also been attempted more or less efficiently but, although each one of these could likely be part of a platform of sensors, none alone can currently provide suitable target detection performance [7].

Among all techniques that are currently under development, ground penetrating radar (GPR) [8] has proven to be one of the few that can provide meaningful operational capabilities [9]. Reports on successful deployment of GPR can be found in [10-13]. This is mainly thanks to the possibility of detecting both metallic and nonmetallic objects together with high-resolution three-dimensional (3D) imaging capabilities $[14,15]$. On the other hand, GPR still suffers from substantial limitations, such as time-consuming acquisitions, as a very dense acquisition is required for a full resolution $3 \mathrm{D}$ image $[16,17]$, and prohibitive false alarm rates. Target detection and discrimination algorithms that perform robustly across different terrain and over many possible objects often require multiple scans. Sensor false alarm rate can be reduced if spatial features and geometrical information can be extracted, and this needs a properly acquired image of the subsurface [18-
21]. The latter weakness is related to the ability to potentially map any dielectric anomaly, which could generate a large number of misleading detections [22]. As demining operations are currently very slow, there is a pressing requirement to develop solutions that can offer significantly higher discrimination performance.

A key to improve performance is to identify, understand and extract the features of the landmine radar signature so that a discriminant plane between the landmine and clutter targets can be identified. A landmine may be characterised by a number of scattering centres, each with its own angular radiation pattern, in particular when the plastic content of the internal structure is high. Most landmines may be considered as multiple layered dielectric cylinders that interact with each other to produce multiple reflections $[23,24]$, and it is expected that these properties can hardly characterise other common cluttered objects.

GPR technology can potentially offer numerous degrees of freedom. The use of multiple polarisations can provide key additional information $[25,26]$, because the response of each polarisation is highly correlated to the landmine geometrical structure as well as to its physical properties $[27,28]$. For example, multiple polarisations have been successfully used to identify different types of targets, such as cables and utilities [29-31], thanks to their explicit polarimetric behaviour, but also to correctly reconstruct complex environments [32, 33]. On the whole, polarisation is supposed and expected to improve the characterisation of the acquired subsurface $[34,35]$.

Polarimetry has been previously applied to landmine detection to acquire the signature of several types of targets [36,37], extract their statistics $[38,39]$ and build learning algorithms and extract geometrical information of the target $[40,41]$.

In previous work [42], the authors have collected free space high range resolution profiles of two explosive-free landmines and have exploited the correlation properties as a function of aspect angle. Results have shown that there is a significant correlation between profiles from different aspect angle, underlining the importance of the features. However, because the main goal of this first investigation was to test the measurement setup and its ability to gather signatures, the analysis was limited to the employment of empty devices under non-operational geometries.

Following the outcomes of the work, the objective of the research activity is to further characterise the landmine signatures 

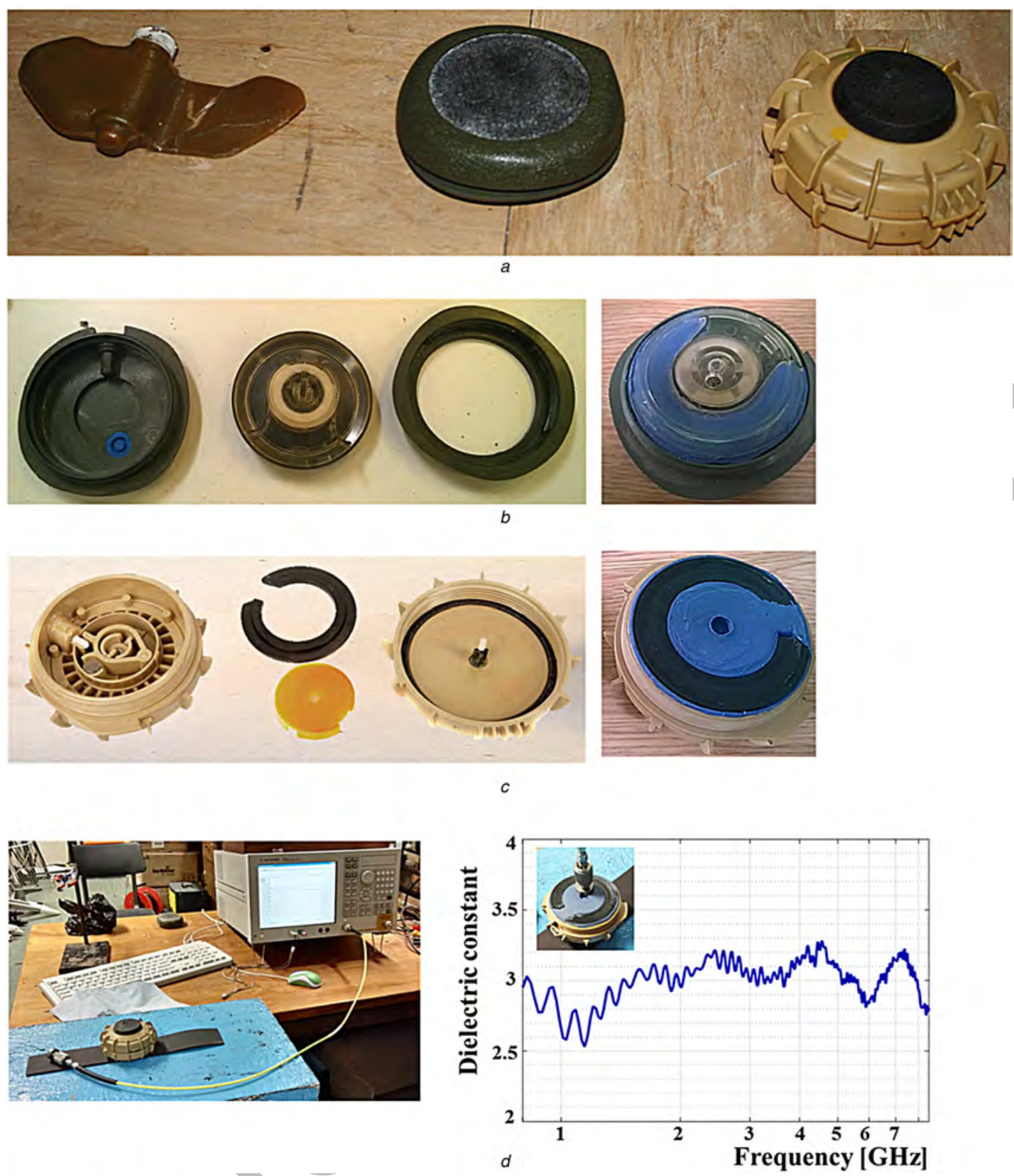

Fig. 1 Target description and details

(a) Pictures of the three employed targets. From left to right: Soviet PFM-1, Italian SB-33 and Italian VS-50 mine, (b) Dismantled targets and filling details, SB-33 landmine, (c) Dismantled targets and filling details, VS-50 landmine, (d) Dielectric characterisation of explosive surrogate sample

in operational geometries as a function of polarisation angle and aspect angle, and identify key and robust features that can be used to enhance detection and discrimination performance.

This paper presents the results obtained by an experimental campaign aimed at collecting the polarimetric range profiles of a number of representative inert landmines, filled with an explosive simulant, at different aspect angles. Effects of polarisation and target inclination angle are evaluated for both off the ground and soil buried devices, with remarkable differences between the two datasets. Buried objects have been investigated with a ground coupled GPR equipment, while a stepped frequency GPR was simulated with a vector network analyser (VNA) for the free space measurements. Consistency between the two experiments has been obtained by maintaining the same central wavelength and hence its ratio with the size of the scatterer. Following this consideration, the operating frequency selected for the free space measurements has been consequently centred on a higher part of the spectrum.

After a detailed analysis, a cross-correlation between profiles is displayed to provide further evidence of the importance of relative geometry between the target and the antennas for signature characterisation.

\section{Target description}

The radar signature of a landmine is highly dependent on the materials used to make the external and internal components as well as the chemical properties of the explosive content. Landmines are objects which are difficult to obtain and replicate to carry out a measurement campaign and therefore it was the first priority to obtain properly constructed inert landmines to ensure the collection of landmine signatures as close as possible to those of a real live device.

Three representative landmines, provided by the Defence Academy of the UK, were used. These were complete with all their external and internal components and were filled with a high explosive simulant commonly used to train the UK Ammunition Technical Officers. A photograph of the three landmines is displayed in Fig. 1a. Two of the landmines were blast antipersonnel landmines, namely the two Italian SB33 and VS50, and one was a scatterable Soviet PFM-1 landmine.

Figs. $1 b$ and $c$ show a section of the SB-33 and VS-50 landmines together with all the internal components and the explosive simulant. The structure of the PFM-1 is such that the landmine cannot be easily opened and hence it was not possible to 
take a picture of its internal components. The physical properties of the landmines are described in Table 1.

Figs. $1 b$ and $c$ highlight the unique internal complexity of this family of targets. The presence of such assemblies is beneficial for detection as these mines, and in general plastic mines, contain significant air gaps to allow movement behind the pressure plate. This affects to some degrees their strength and the features of their signature. For this reason, the possibility of detecting internal reflections or scattering from multiple assemblies could represent an important key point for target discrimination.

As opposed to the latter two models described in Table 1, the PFM-1 device does not present any internal components and cannot be considered as a superimposition of dielectric cylinders. However, it plays a fundamental role for investigating devices with a distinct structure, different complexity and internal design to provide a more straightforward comparison.

The permittivity of the filling material has been measured, to validate the expected electrical and chemical adherence to typically employed explosives, with an Agilent 85070E Dielectric Probe kit carrying a coaxial probe operating from $200 \mathrm{MHz}$ to $20 \mathrm{GHz}$ [43]. Fig. $1 d$ shows the setup and the results of the dielectric measurements taken on the substance sample. These can be compared with the typical values of commonly employed explosive listed in Table 2.

Although the PFM-1 contains liquid plastic explosive with higher dielectric values, the expected differences in the signature are limited to a variation in magnitude and therefore their effect on the scope of this investigation can be ignored.

\section{Free space experimental trial}

To exploit the landmine signature variations with acquisition geometries, a set of free space measurements have been carried out at the Defence Academy of the UK.

Data were collected using a MS46322A Anritsu VNA transmitting a stepped frequency waveform with a bandwidth of $3.5 \mathrm{GHz}$ from 5 to $8.5 \mathrm{GHz}$.

Although the employed frequency band would allow very limited soil penetration for subsurface imaging, it was selected to obtain a typical value of the ratio between common propagating wavelengths in the ground and the size of the landmine. A central wavelength of $4.6 \mathrm{~cm}$, in free space, corresponds to a frequency of $6.5 \mathrm{GHz}$, and this value of the wavelength is used to compute a hypothetical downshifted system for typical soil characteristics.

In particular, a $4.6 \mathrm{~cm}$ wavelength corresponds to a system with a central frequency of $2.4-3.2 \mathrm{GHz}$ in dry sandy or loamy soil $(\varepsilon$ : $4-7)$ and $1.5-2.4 \mathrm{GHz}$ in wet soils ( $\varepsilon: 9-20)$.

Considering that mostly GPR equipment employed in demining operations works in a frequency range from 1 to $3 \mathrm{GHz}$, the achieved equivalence corresponds to a realistic operational configuration (MINEHOUND [44], ALIS [45], HSTAMIDS [46], NIITEK [47]).

Another consideration is that air is a less dense material with a very low absorption rate, compared with typical encountered soils. This will lead to a better characterisation of the signature features, as all the expected multiple reflections coming from the different

Table 1 Targets description

\begin{tabular}{lcc}
\hline Device & Length/width/height, $\mathrm{mm}$ & Weight, $\mathrm{g}$ \\
\hline PFM-1 & $120 / 20 / 61$ & 75 \\
SB-33 & $85 / 85 / 30$ & 140 \\
VS-50 & $90 / 90 / 45$ & 185 \\
\hline
\end{tabular}

Table 2 Dielectric constant of explosive substance [8]

\begin{tabular}{lc}
\hline Material & Value \\
\hline TNT & 2.70 \\
RDX & 3.14 \\
Comp B & 2.90 \\
PETN & 2.72 \\
Semtex H & 3.00 \\
\hline
\end{tabular}

assemblies of the target will likely be effectively recorded. The effects on polarisation are such that the soil will have an impact in the presence of several heterogeneities, but homogeneous soil will not alter the wave characteristics [48].

Two identical horn antennas in quasi-monostatic configuration and parallel polarisation were mounted on a LinearX precision turntable to collect polarimetric range profiles with a $5^{\circ}$ rotation step over $180^{\circ}$. The turntable was mounted on the vertical face of a L-shaped metallic frame to ensure a perpendicular alignment with respect to the ground. The antennas were arranged to transmit and receive with the same polarisation and rotating the turntable allowed measurements of the targets with different polarisation angles (i.e. different angles of the incident linear E-field with respect to the landmine). Fig. $2 a$ shows the antenna geometry.

A summary of the experimental activity is provided in Table 3 .

The landmines under test were placed at a distance of $\sim 170 \mathrm{~cm}$ from the antennas plane on a styrofoam cone. The experimental setup is presented in Fig. $2 b$.

The styrofoam material was used due to its low reflection properties to minimise the impact of the stand. A measurement of the background was taken to remove all stationary clutter from the target signature.

Results for all targets are analysed in detail at two different aspect angles to further quantify the impact of target inclination on the signature. The geometry is shown in Fig. $2 c$.

The choice of evaluating the radar signature at different target angle is motivated by the fact that being a composite target with a number of internal scatterers, landmine response could provide different features and characteristics. In a large variety of environments, landmines may have been subject to alterations, such as landslips and flooding, which may have modified the geometry and orientation of the buried target.

Each signature has been normalised to its own maximum value to help the comparison process and displayed as range profiles in the time domain. As an additional evaluation element, the correlation coefficients between profiles have been computed to further highlight reflections consistency. The correlation matrix

$$
\boldsymbol{R}(x, y)=\left[\begin{array}{ll}
\rho(x, x) & \rho(x, y) \\
\rho(y, x A) & \rho(y, y)
\end{array}\right]
$$

has been computed for each pairwise profile combination, on the mean-adjusted and standard deviation normalised profiles, with $\rho$ representing the Pearson coefficients, calculated as

$$
\rho(x, y)=\frac{1}{N-1} \sum_{i=1}^{N}\left(\frac{x_{i}-\mu_{x}}{\sigma_{x}}\right)\left(\frac{y_{i}-\mu_{y}}{\sigma_{y}}\right)
$$

\subsection{Results and discussion}

The effect of antenna geometry on the PFM-1 mine as a function of aspect angle is presented in Fig. 3 together with the computed correlation analysis.

As expected, the polarimetric behaviour of the target is almost constant due to its relatively simple structure. There is a main scattering contribution in the range of the target which is overall regular also with aspect angle. When the target is inclined (Fig. $3 b$ ), the effects of antenna polarisation become slightly evident from some weak variations due to the different illumination of the target.

The correlation coefficients in Figs. $3 c$ and $d$, computed from the cross-correlation function between profiles collected at different polarisation for each inclination angle, validate the previously made considerations. There is a very high level of correlation among profiles, especially when the target is placed at zero inclination angle (Fig. $3 c$ ). The variations detected in the signature when the target is laterally rotated are confirmed, as the areas around the orthogonal polarisation show slightly lower values, meaning that there are some differences (Fig. 3d). However, on average, the polarimetric analysis shows high levels of correlation above 0.8 . 


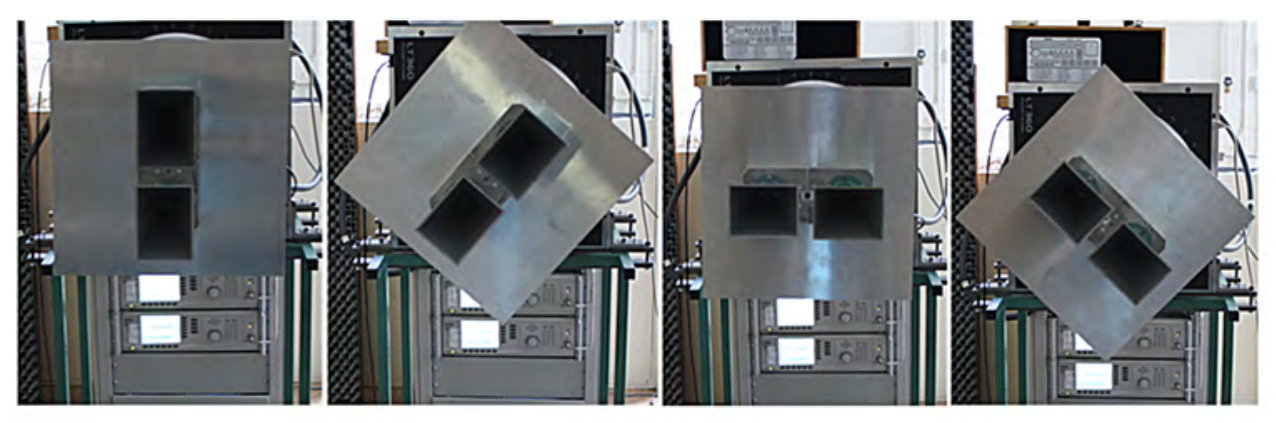

a

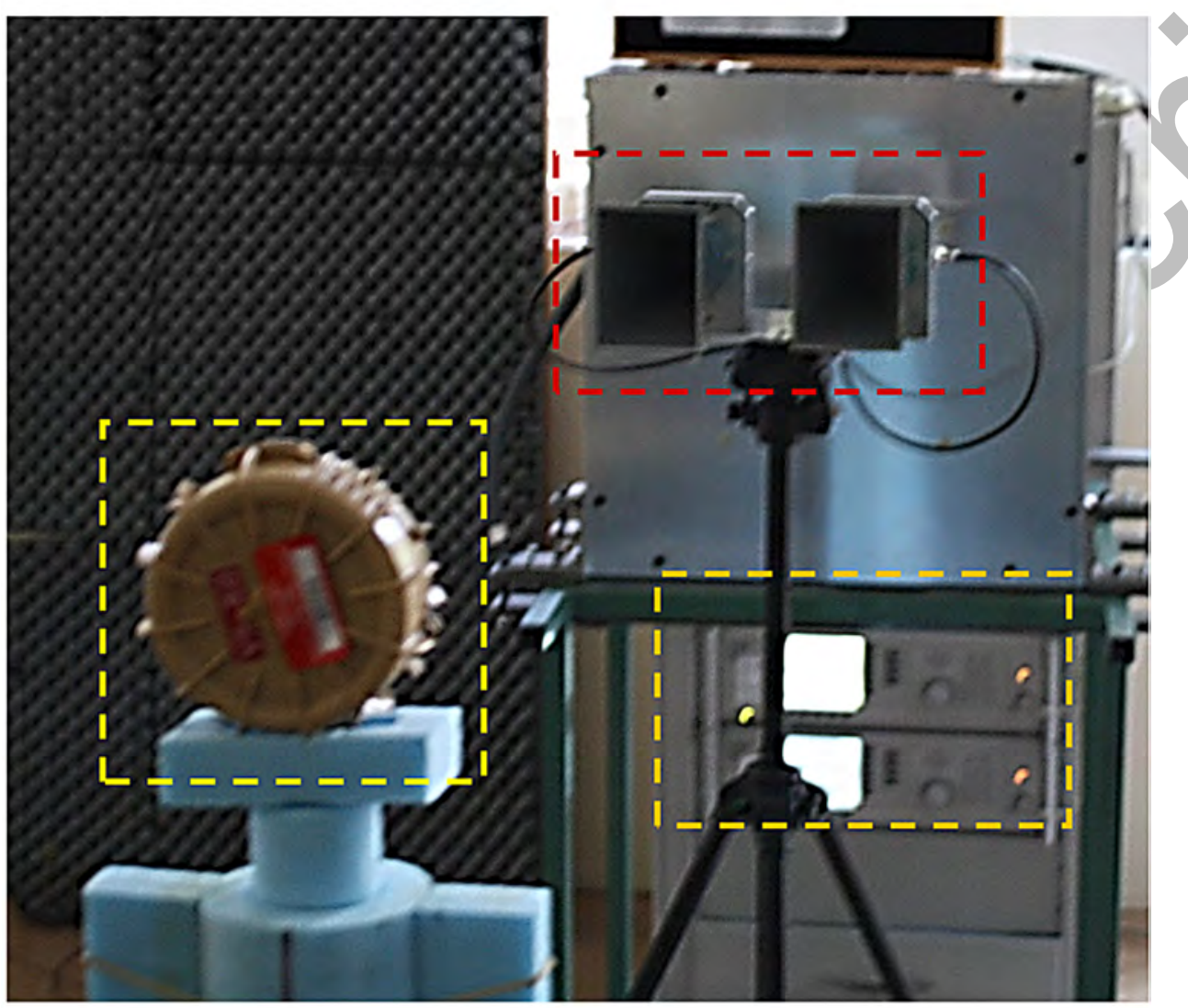

b
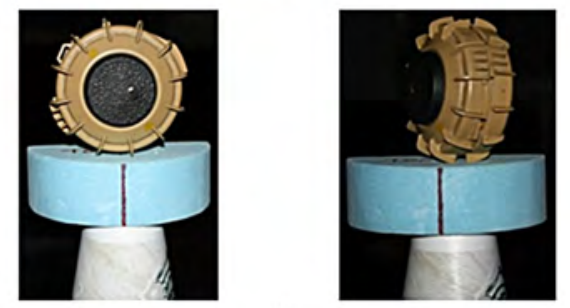

Fig. 2 Free space measurements details, data acquisition

(a) Data acquisition, as a reference, the positive direction of rotation is clockwise. From left to right: starting polarisation (HH), $45^{\circ}$ orientation, orthogonal polarisation (VV), $135^{\circ}$ inclined orientation, (b) Experimental setup with the two horns connected to the VNA and facing the landmine under test on the stand, (c) Target aspect angles: left $0^{\circ}$, right $45^{\circ}$

The polarimetric profiles for the SB-33 mine are presented in Fig. 4.

In this case, the collected measurements show a higher level of behaviour and features, as the radar response is an image of the complexity of the device. The SB-33 has a larger physical dimension than the PFM-1, hence it is quite obvious that its response when the target is placed at no inclination angle (Fig. $4 a$ ) will be thicker in space, but what is to be noticed is that the main contributions is not constant, but some variations in the magnitude of the peaks occur. This feature is a suggestion of the presence of inner assemblies which gives rise to multiple reflections.

The effect is even more evident when the target creates an angle towards the antennas plane (Fig. $4 b$ ), in which both reflection
Table 3 Free space measurements experimental setup

\begin{tabular}{lc}
\hline Parameter & Value \\
\hline frequency range, $\mathrm{GHz}$ & $5-8.5$ \\
frequency step, $\mathrm{MHz}$ & 0.4375 \\
central wavelength, cm & 4.6 \\
angular range, deg & $0-180$ \\
antenna dimension, cm & $9 \times 12$ \\
antenna offset, cm & 9
\end{tabular}




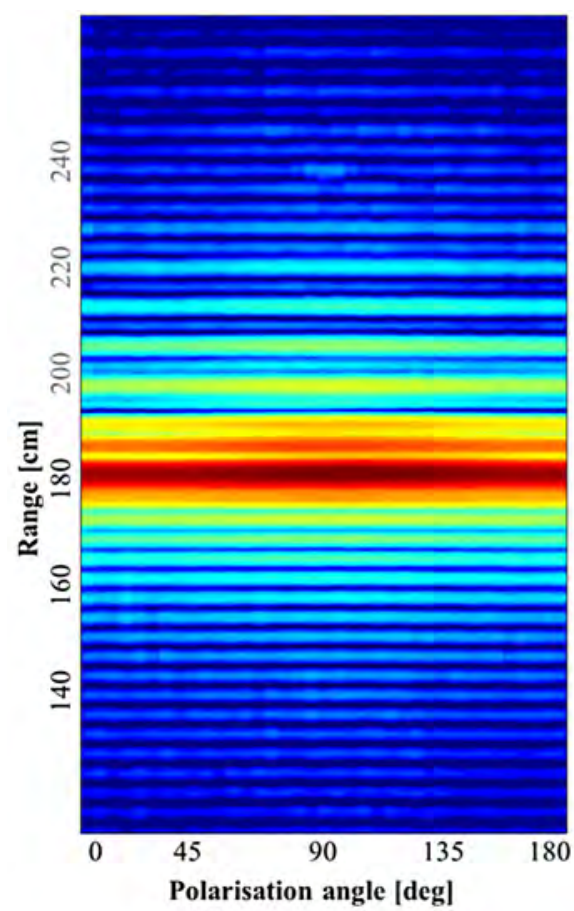

a

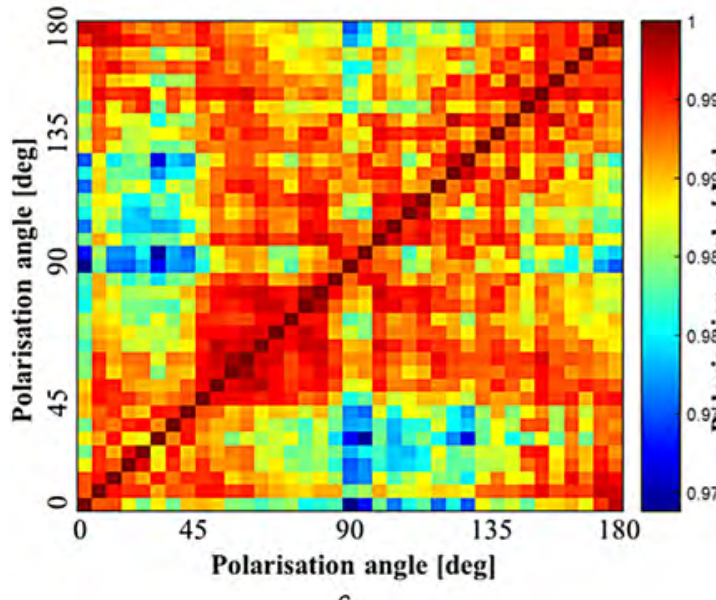

$c$
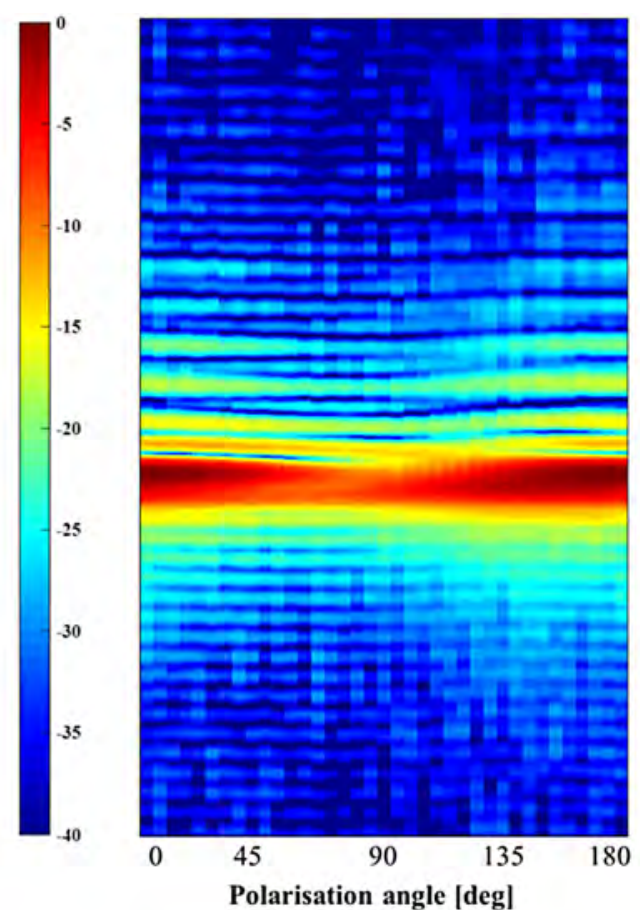

b

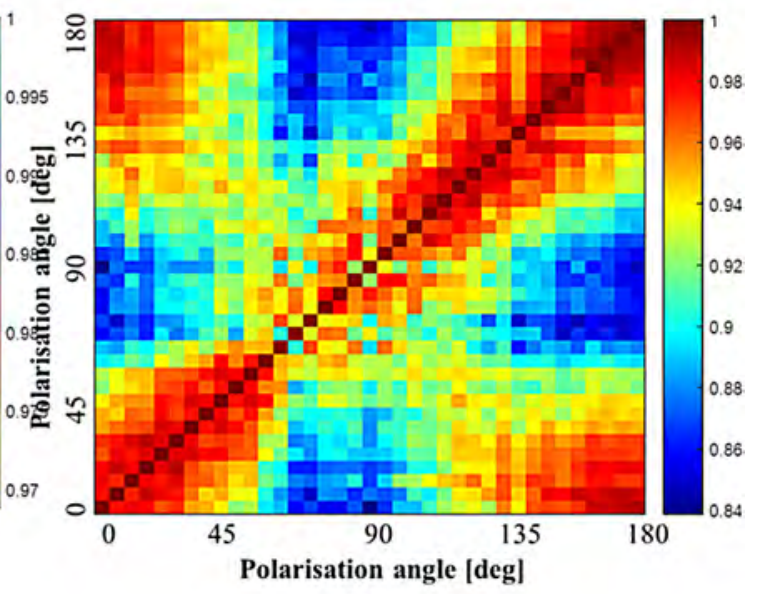

Fig. 3 Free space polarimetric profiles and correlation analysis of the PFM-1 landmine. Aspect angles (a) $0^{\circ}$ signature, (b) $45^{\circ}$ signature, (c) $0^{\circ}$ correlation values, (d) $45^{\circ}$ correlation values

population and distribution suffer significant variations with polarisation angle. Therefore, for composite targets, polarisation seems to be able to provide valuable information on the target.

Just from the first view of the correlation analysis, it is clear how the internal structure of the landmine impacts the polarimetric response. In all the frames, the signatures decorrelate very fast, as there is a sharp transition between the main diagonal and the surrounding coefficients. While when the target is at $0^{\circ}$ (Fig. $4 c$ ), the average values is still over 0.8 , for the other matrix (Fig. $4 d$ ) the values reduce up to $20 \%$.

Fig. 5 presents the acquired profiles for the VS-50 mine.

The same considerations made for the SB-33 mine hold here, as the internal structure affects the polarimetric trend in a clear and noticeable way. The profile in Fig. $5 a$ is less heterogeneous comparing to its SB-33 equivalent due to the presence, just below the activator plate, of a large number of air gaps, which modify the signature and balance out the illumination changes. When these gaps are not dominant over the signature, when the target is rotated (Fig. $5 b$ ) the profiles return to describe a more complex polarisation dependent behaviour.

Due to the underlined internal complexity, the correlation coefficients describe a situation in which significant variations between profiles occur. For the geometry of Fig. $5 c$, signatures are almost homogeneous, demonstrating the assumptions on the air gaps effects. When the target does not look to the antennas, instead, the correlation between profiles gets lower, as the effect of the void becomes not dominant.

\section{Field measurements}

To validate the highlighted features and to provide a more realistic trial environment, the same acquisition were carried out burying the same landmines in a sand pit. The three targets were buried at a depth of $\sim 10 \mathrm{~cm}$.

Due to humidity, the sand was not completely dry, providing a relative dielectric constant of $\sim 9$ and a consequential velocity of $10 \mathrm{~cm} / \mathrm{ns}$. Referring to the previous consideration for the selection of the operating frequency of the free space measurements, the equivalent system should have been centred at a frequency of 6.5 $\mathrm{GHz}$ to maintain the same wavelength to target size ratio.

The employed equipment was an IDS Aladdin (IDS Georadar srl) georadar platform, a shielded ground coupled dipole antenna, spaced $9 \mathrm{~cm}$, with a central frequency and bandwidth of $2 \mathrm{GHz}$. These parameters give a central wavelength of $0.05 \mathrm{~cm}$, therefore a high consistency with the previously described experiments has been successfully achieved.

Accurate rotation was performed by a mechanical turntable (Fig. 6a). 


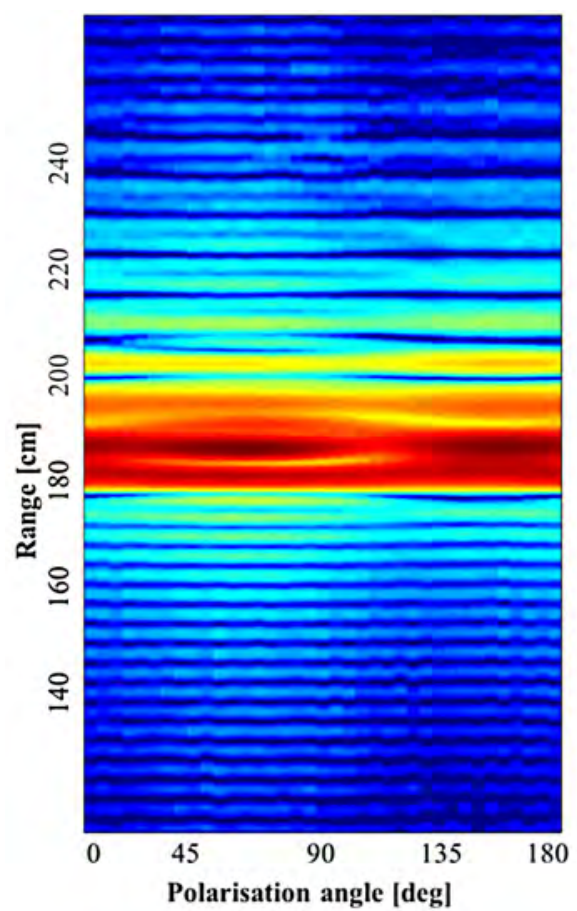

a

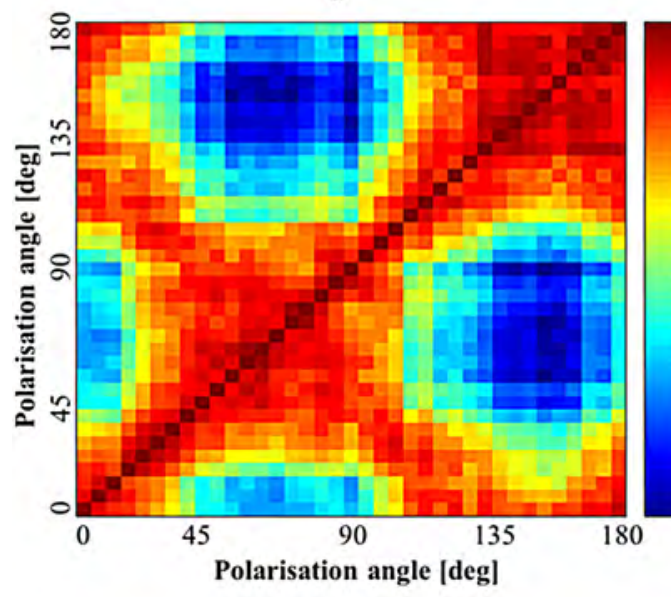

$c$
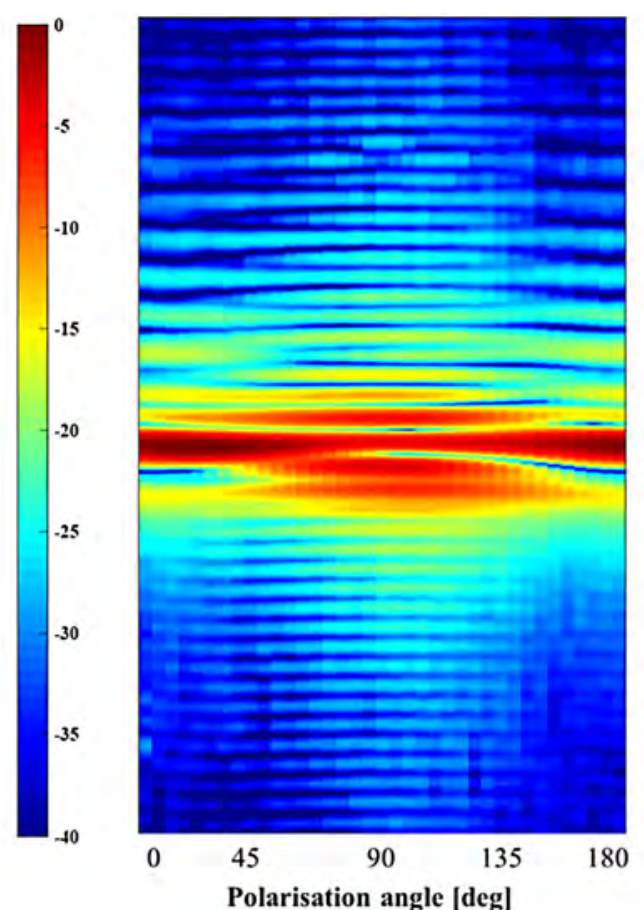

b

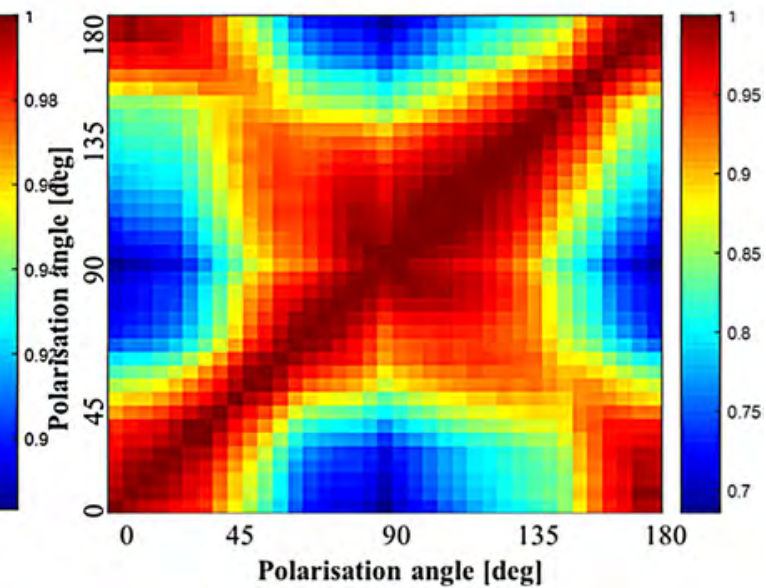

d

Fig. 4 Free space polarimetric profiles and correlation analysis of the SB-33 landmine. Aspect angles

(a) $0^{\circ}$ signature, (b) $45^{\circ}$ signature, (c) $0^{\circ}$ correlation values, (d) $45^{\circ}$ correlation values

As shown in Fig. 6, the targets were buried in sharp sand, with very low clay content and gritty texture for a better drainage. This last aspect was fundamental to avoid trench effects when burying the target in humid conditions.

Data were collected with the reflection centre of the antenna right in the middle of the target and following the previous strategy: a $180^{\circ}$ rotation, with an angular sampling of $5^{\circ}$. The experimental setup is detailed in Table 4 and Fig. $6 b$.

The processing chain applied to the data [49] consisted of a linear frequency filtering and a spherical exponential compensation gain function, matched to the soil characteristics, to recover the amplitude losses. The correlation analysis has been computed as well, following the previous indications.

\subsection{Results and discussion}

The PFM-1 landmine results, depending on the antenna orientation and aspect angle are shown in Fig. 7.

As expected, due to the soil absorption, the signature presents lower information content than the free space equivalent (Fig. 3). A single reflection is detectable when the target is placed at an aspect angle of $0^{\circ}$, with a polarimetric trend due to the presence of the cylindrical fuze well, behaving as metallic linear targets. This difference from the free space measurements is likely be a consequence of the larger pattern of the dipole antennas, which is dominated by the presence of the metallic assembly. When the target is rotated (Fig. $7 b$ ) two events can be clearly identified, corresponding to the first reflection coming from the upper surface of the landmine, and the second one, related to the bottom interface. A consistent trend with the free space trial can be noticed, as the signature intensity decreases in the range $45-90^{\circ}$, with a lower magnitude.

Correlation analysis (Figs. $7 c$ and $d$ ) shows consistent trends and values, with higher values for the horizontally laying configuration, due to the lower level of information content in the signature.

Fig. 8 describes the results from the analysis of the SB-33 device.

The same consideration can be outlined for the second objects, characterised with a highly heterogeneous and composite design, with some exceptions. A single reflection is visible when the target is oriented at $0^{\circ}$ towards the antennas, with nothing related to the internal structure. This effect can be verified with the higher correlation values of the corresponding Fig. $8 c$. A rotation of the target produces a significant response, as three well-defined events have been recorded. These belong to the upper surface, probably to the air gaps inside the landmine or the fuze assemblies (refer to Fig. $1 b$ for the structure of the SB-33) and the bottom reflection, respectively. The latter reflection, obviously, has almost half of the magnitude of the other two, with the air interface being the higher 

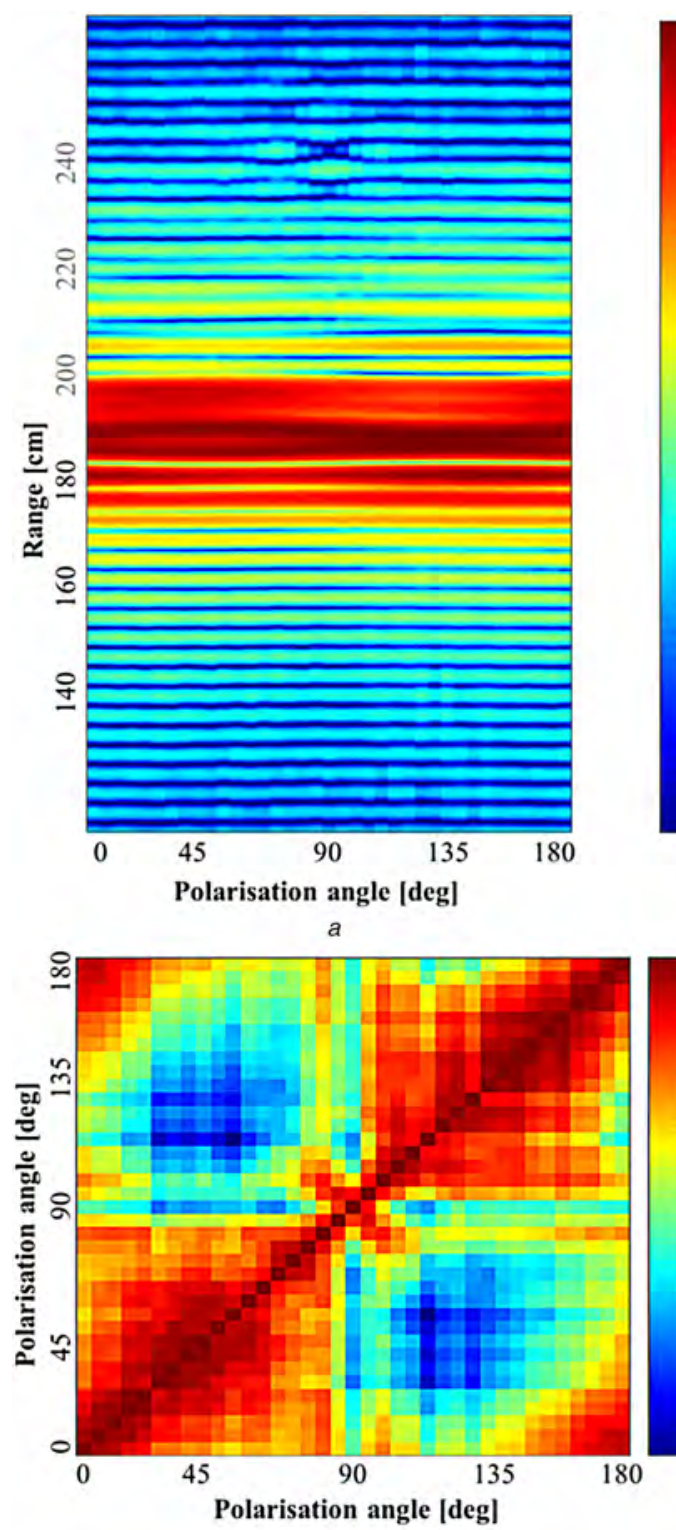

$c$
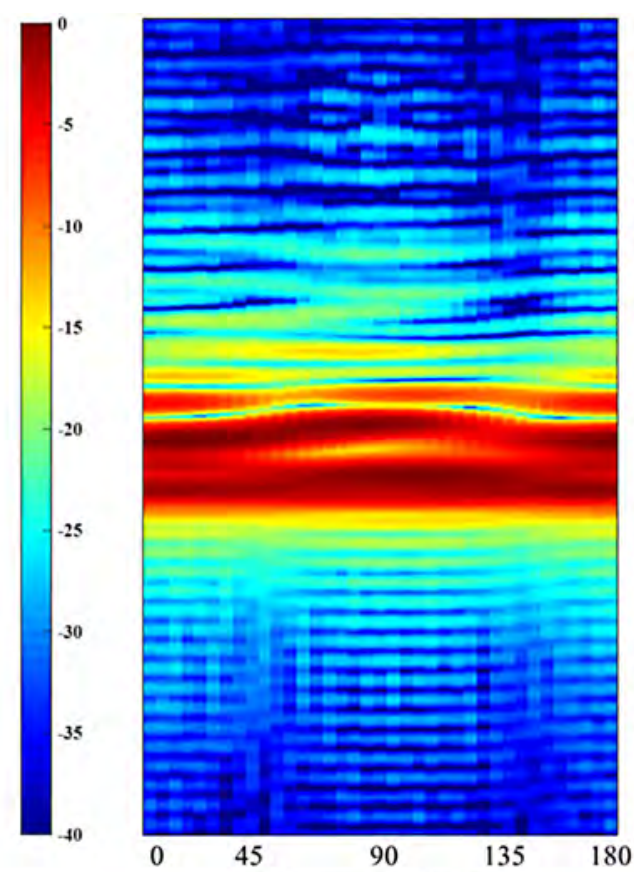

Polarisation angle [deg]

$b$

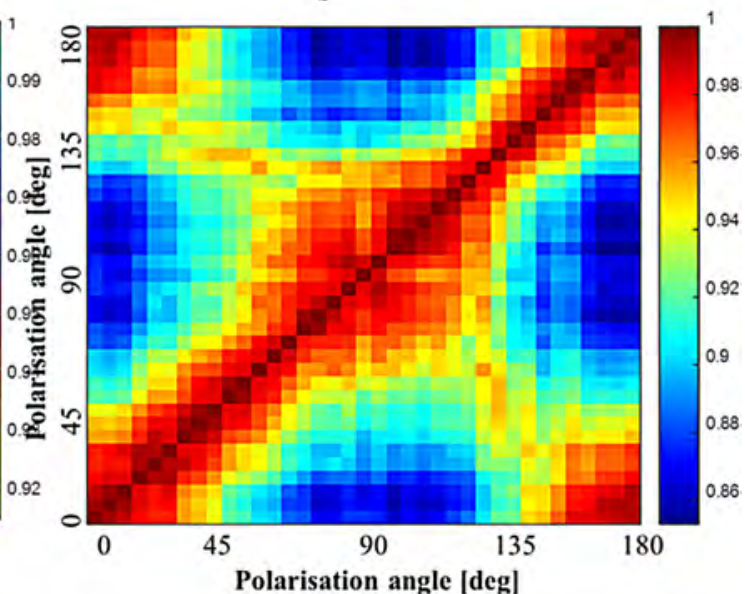

d

Fig. 5 Free space polarimetric profiles and correlation analysis of the VS-50 landmine. Aspect angles

(a) $0^{\circ}$ signature, (b) $45^{\circ}$ signature, (c) $0^{\circ}$ correlation values, (d) $45^{\circ}$ correlation values

and more stable one. These multiple scattering was visible when measuring the target in air (Fig. 8b), even if the presence of the internal reflections complicated the identification of the three effects.

Signatures of the VS-50 are presented in Fig. 9.

The investigated device has an internal design (Fig. 1c) characterised by the presence of a sunburst-shaped air gaps just below the activator plate: this is clearly visible when this assembly is directly below the GPR platform (the stronger reflection in Fig. $9 a$ ). Oppositely to the signature of the SB-33, in this case the number of detectable interfaces is more for an aspect angle of $0^{\circ}$ than with an inclined target. This is due to the presence of the described air layer that becomes of secondary importance when the target is rotated, while the SB-33 has a bulk of air located deeper into the structure, hence mostly dominant when the landmine is inclined.

Table 5 provides a comparison based on average and standard deviation of the correlation coefficient for each of the presented experiments.

Although being basic statistical descriptors, these two values are a convenient instrument to compare the consistency of the results. The values in the table summarise what has been commented, and while the average value could be affected by outliers and very similar signature, the deviation of the correlation coefficients represents their maximum fluctuation, therefore it is a significant descriptors for evaluating the variations of the signature over the polarisation space.

The offset between the free space measurements and the field one is a consequence of the lower level of details and information of the signatures that will remove a certain amount of continuity among recorded data. The outcome is twofold and with opposite behaviour depending on the structure of the landmine: for composite targets, the inability of the system to detect scattering from internal assemblies will increase the correlation values and their continuity; for objects that does not present internal components, as the PFM-1 for instance, this will lower the correlation.

\section{Conclusion and development}

The carried out experiments have demonstrated that geometry plays an important role when investigating composite targets. As landmines are generally characterised by a complex design, both in terms of outer casing and internal assemblies, the different angular pattern described by these different structures has a significant impact on the radar signature of these devices. The real challenge is to be able to proper record these scattering features and correctly determine the nature of the detected targets.

Through an ensemble of free space measurements of three different representative inert landmines, explosive filled and 

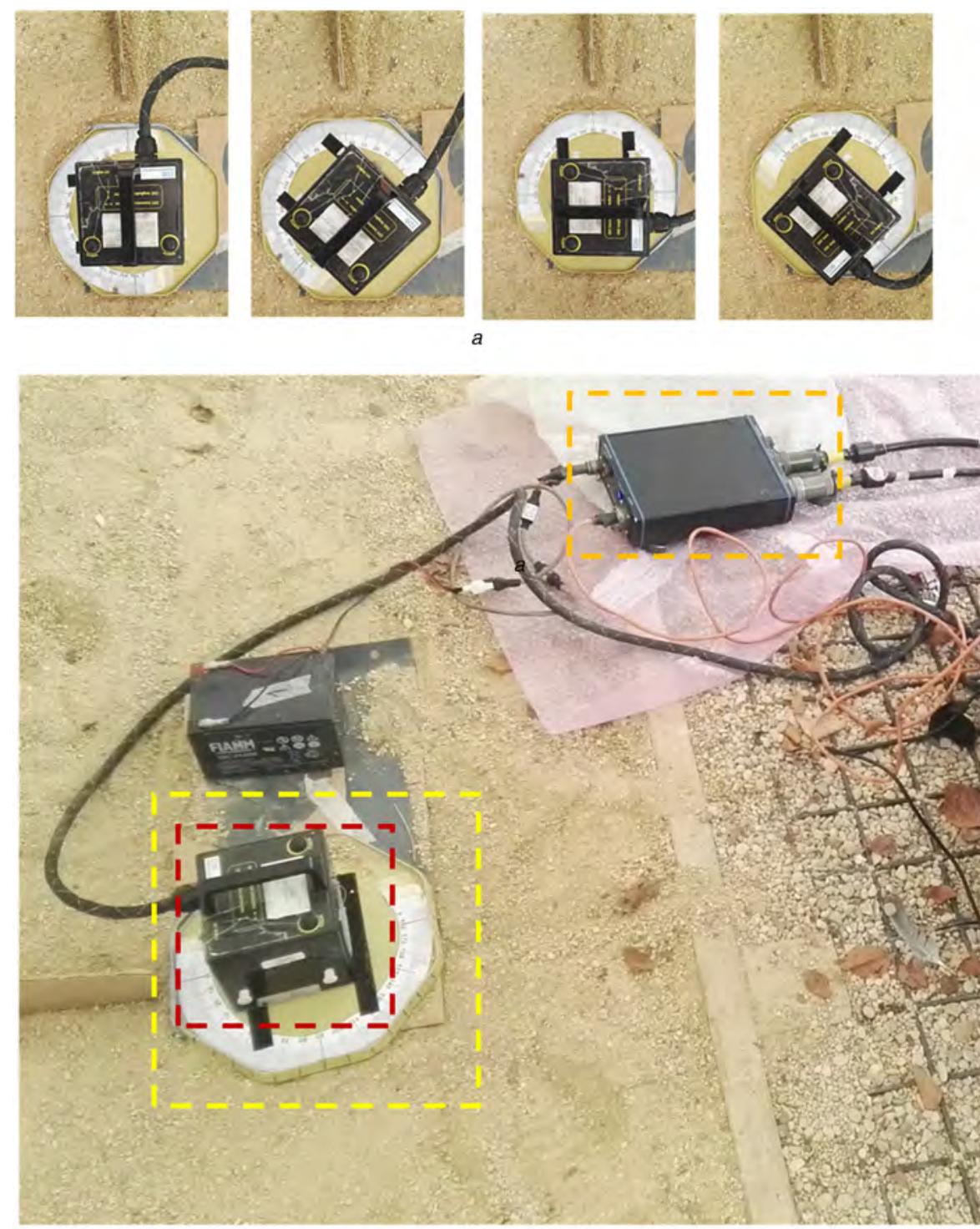

$b$
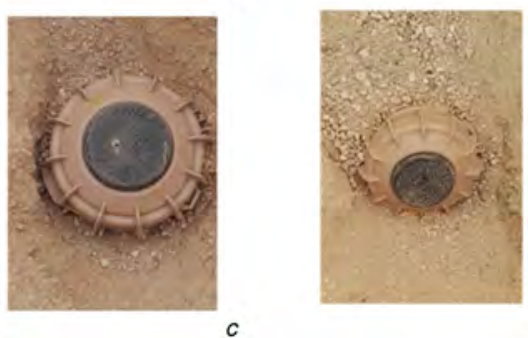

Fig. 6 Field measurements details, data acquisition

(a) Data acquisition, as a reference, the positive direction of rotation is clockwise. From left to right: starting polarisation $(\mathrm{HH}), 45^{\circ}$ orientation, orthogonal polarisation (VV), $135^{\circ}$ inclined orientation, (b) Experimental setup with the GPR platform connected to the central unit and over the mechanical turntable, (c) Target aspect angles: left $0^{\circ}$, right $45^{\circ}$

Table 4 Field measurements experimental setup

\begin{tabular}{lc}
\hline Parameter & Value \\
\hline frequency range, $\mathrm{GHz}$ & $1-3$ \\
frequency sampling, $\mathrm{GHz}$ & 17 \\
central wavelength, $\mathrm{cm}$ & 5 \\
angular range, deg & $0-180$ \\
antenna offset, cm & 9 \\
time window, ns & 20 \\
\hline
\end{tabular}

complete of all their design parts, the paper has demonstrated the importance of polarisation for determining the heterogeneity of the target, as inner assemblies do not behave as the other parts of the mine, thus a change in the illumination pattern is sufficient, in some case, to make these aspects visible, providing deeper information on the exploited object.

A further element that has proven to be valuable of exploitation is the relative geometry between the target and the antennas, namely inclination or aspect angle. Its importance rises from the fact that, considering the compositeness of these devices, depending on the facet which is pointing at the antenna the contribution to the overall signature will vary. The VS-50, which has several air gaps just below the activator plate, is a clear demonstration of this, as the presence of void is prevailing over other scattering effects, reducing the polarimetric effects.

The situation changes when investigating buried targets, as the absorption effects significantly alter the level of details and information gathered by the signature analysis. A confirmation trial was carried out with the same targets buried in sharp sand and acquired with a ground coupled GPR platform. The frequency 

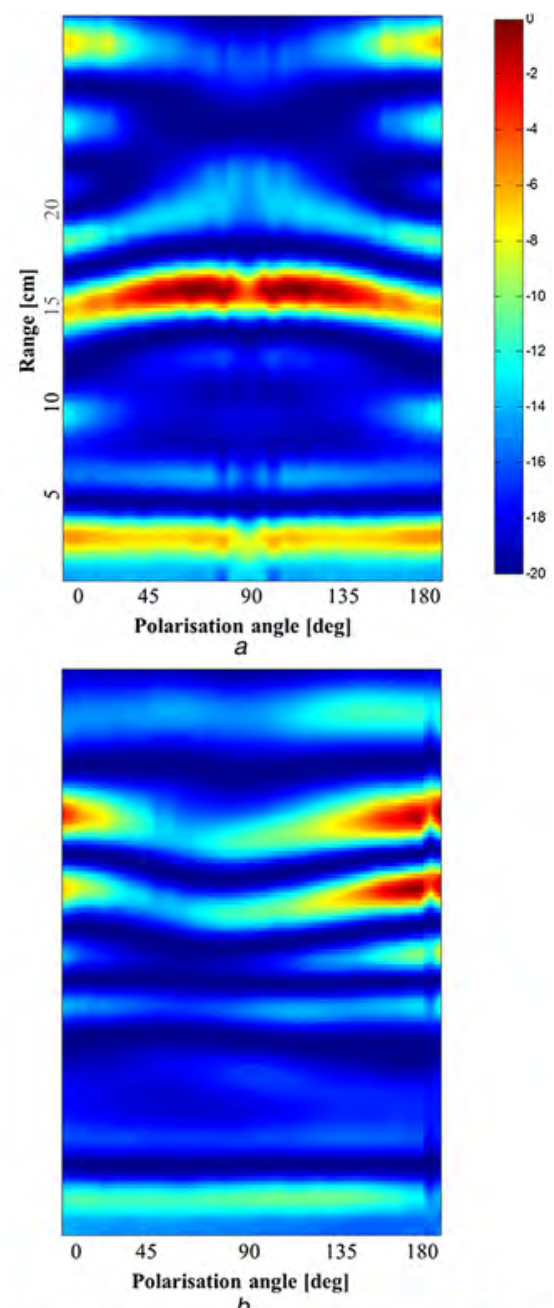

b
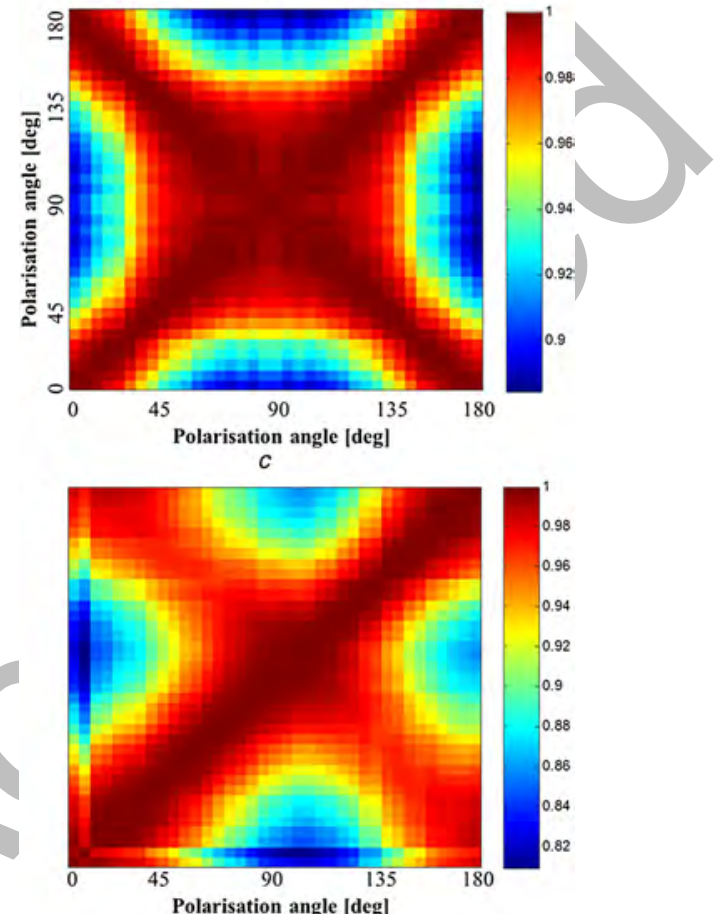

$d$

Fig. 7 Soil buried polarimetric profiles and correlation analysis of the PFM-1 landmine. Aspect angles

(a) $0^{\circ}$ signature, (b) $45^{\circ}$ signature, (c) $0^{\circ}$ correlation values, (d) $45^{\circ}$ correlation values

ranges of the two experiments were chosen to maintain the ratio between the propagating wavelength and the size of the landmine.

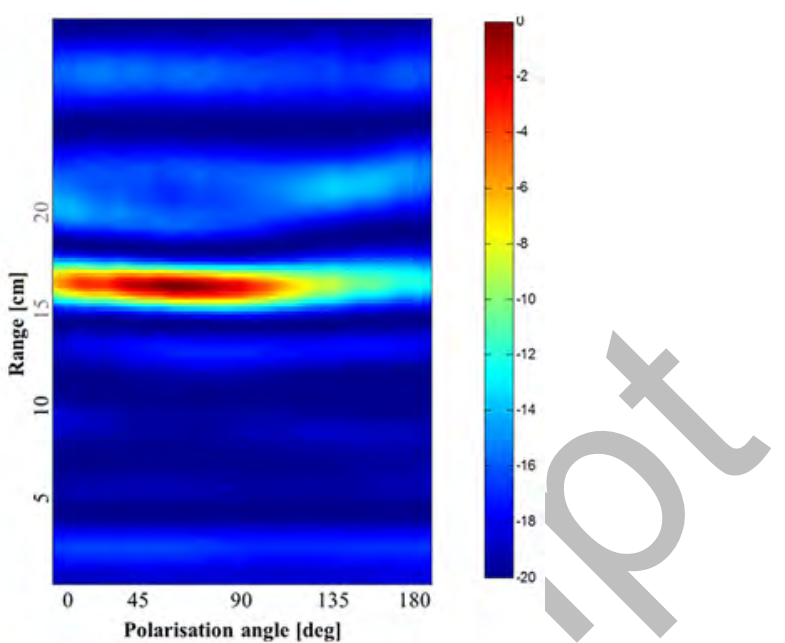

a
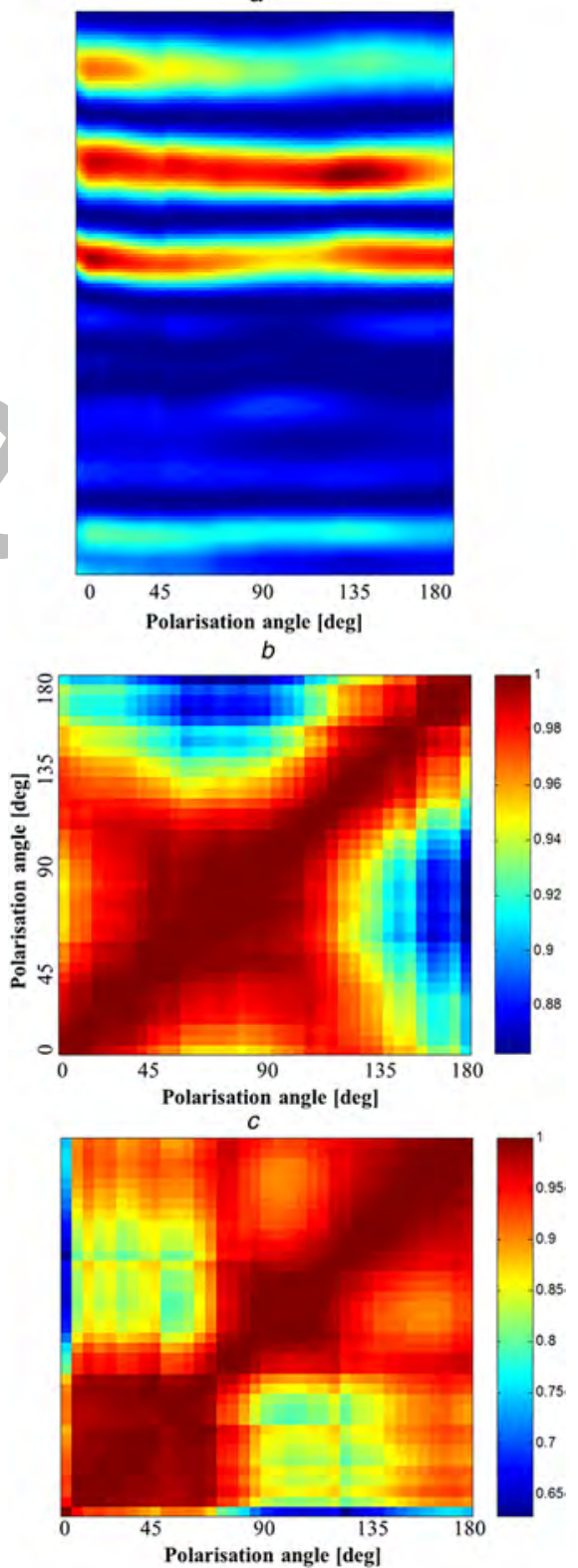

Fig. 8 Soil buried polarimetric profiles and correlation analysis of the SB-33 landmine. Aspect angles

(a) $0^{\circ}$ signature, (b) $45^{\circ}$ signature, (c) $0^{\circ}$ correlation values, (d) $45^{\circ}$ correlation values

A comparable trend was found, as the spatial distribution and location of the main reflections were consistent between the two trials. Huge differences have been noticed in the density of the reflections for the soil buried targets. While in free space the 

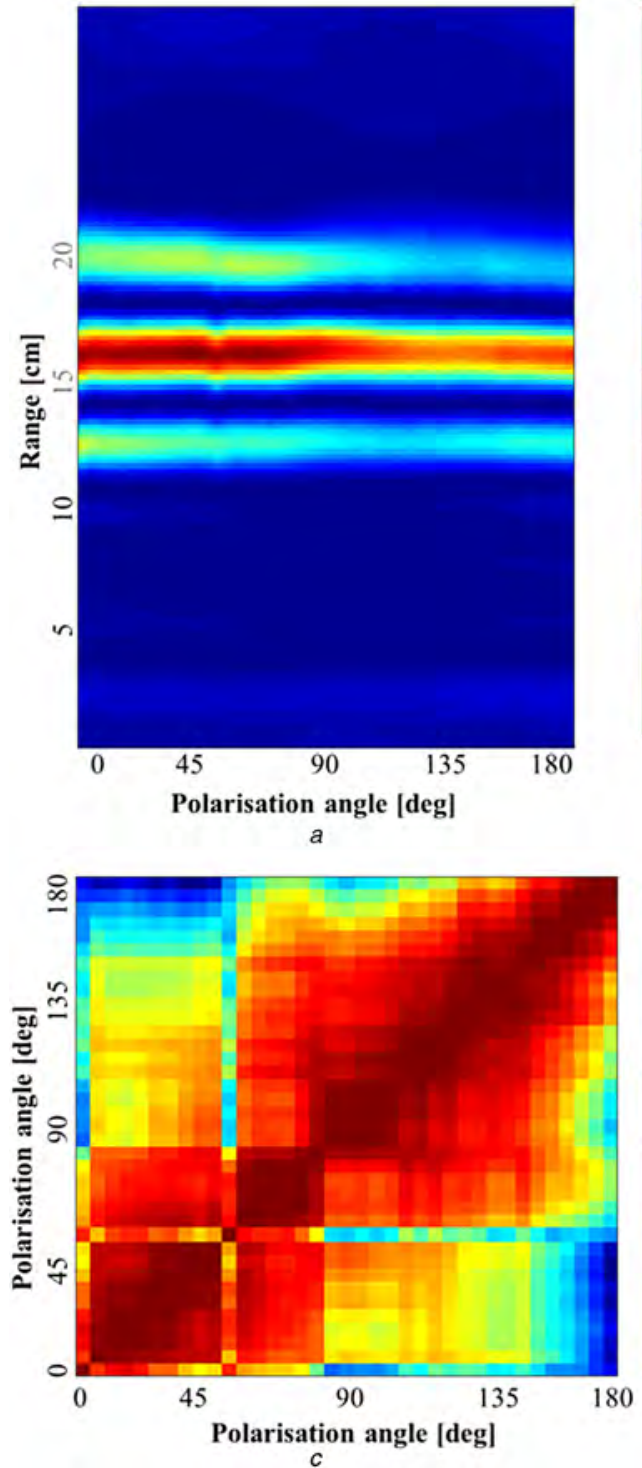
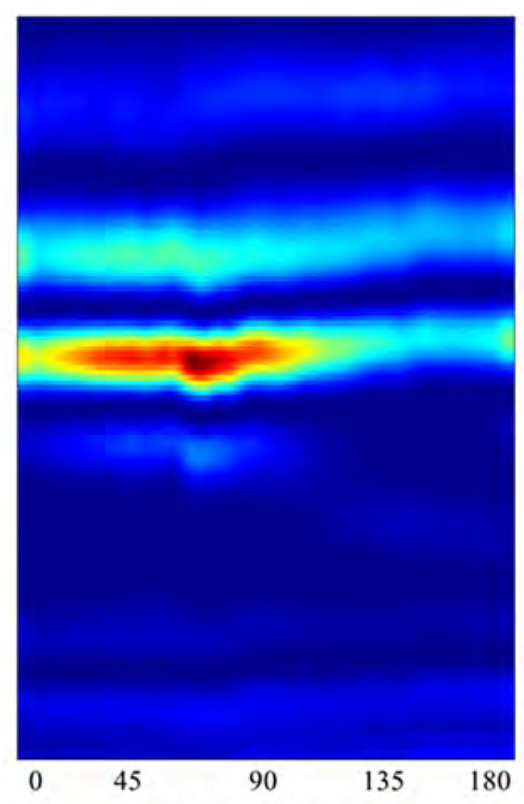

Polarisation angle [deg]

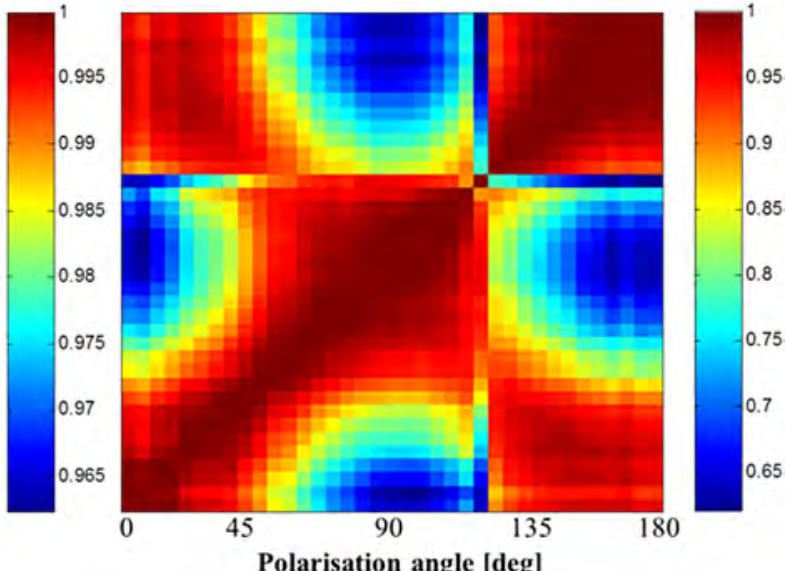

Fig. 9 Soil buried polarimetric profiles and correlation analysis of the VS-50 landmine. Aspect angles (a) $0^{\circ}$ signature, (b) $45^{\circ}$ signature, (c) $0^{\circ}$ correlation values, (d) $45^{\circ}$ correlation values

Table 5 Statistical comparison

\begin{tabular}{lccccc}
\hline Target & Aspect angle & \multicolumn{2}{c}{ Free space } & \multicolumn{2}{c}{ Sand pit } \\
& & Average & Deviation & Average & Deviation \\
\hline PFM-1 & $0^{\circ}$ & 0.99 & 0.005 & 0.9776 & 0.0214 \\
& $45^{\circ}$ & 0.93 & 0.038 & 0.8396 & 0.1430 \\
SB-33 & $0^{\circ}$ & 0.95 & 0.031 & 0.9733 & 0.0243 \\
& $45^{\circ}$ & 0.88 & 0.085 & 0.9206 & 0.0693 \\
VS-50 & $0^{\circ}$ & 0.96 & 0.019 & 0.9899 & 0.0085 \\
& $45^{\circ}$ & 0.93 & 0.040 & 0.8784 & 0.1149 \\
\hline
\end{tabular}

internal assemblies and structures were visible, burying the target into a lossy ground allowed nothing but the strongest reflections to be successfully collected at the surface. In particular, internal reflections are detectable only for the VS-50 and the SB-33, due to the presence of a relatively large air gap inside the structure and only in favourable geometrical conditions.

Having understood the significance of the investigated parameters, several consequent developments could be highlighted.

First of all, it is fundamental to characterise the polarimetric behaviour of clutter targets. Hence, the same acquisition and processing scheme should be applied to targets that are a common source of false alarm, such as stones and roots, as well as battlefield debris. The aim is whether to confirm or not the suitability of polarisation to be a valuable parameter for increasing GPR performance.
Future work will also include an investigation on the impact that heterogeneous soil has on the highlighted behaviour, as it is known that certain terrain conditions could depolarise the wave and hence the polarimetric information would be significantly different.

In addition, employed landmines were almost in their original conditions, therefore another valuable analysis would be the investigation of the weathering effects on the device, to determine whether a landmine at some stage of its burial will provide the same features or there are any scattering variations. This is of particularly interest as most of landmines have been buried for decades and subject to a large number of natural and artificial phenomena. 


\section{Acknowledgments}

The authors thank the Find A Better Way charity for their support of this research under the DETERMINE programme (grant number 2015/001D). They also thank the Defence Academy Ammunition Hall for providing the real landmines used for the experiments.

\section{References}

[1] 'International Campaign to Ban Landmines'. Available at http://www.icbl.org

[2] 'UN News Centre'. Available at http://www.un.org/apps/news/story.asp? NewsID $=54736$

[3] 'The Guardian'. Available at https://www.theguardian.com/globaldevelopment/2016/apr/04/ukraine-attempt-defuse-landmines-as-more-areplanted

[4] 'Landmine and Cluster Munition Monitor'. Available at www.themonitor.org

[5] Bier, G.L.: 'The economic impact of landmines on developing countries', Int. J. Soc. Econ., 2003, 30, (5), pp. 651-662

[6] Acheroy, M.: 'Mine action: status of sensor technology for close-in and remote detection of anti-personnel mines', Near Surf. Geophys., 2007, 5, (1), pp. $43-55$

[7] Bruschini, C., Bertrand, G.: 'A survey of research on sensor technology for landmine detection', J. Conv. Weapons Destr., 2016, 2, (1), pp. 1-25 Daniels, D.: 'Ground penetrating radar' (IEE, 2004)

[9] Sahli, H., Bruschini, C., Crabbe, S.: 'Catalogue of advanced technologies and systems for humanitarian demining', EUDEM2 Technology Survey, 2005. 1.3, pp. 8-35

[10] Sato, M., Fujiwara, J., Kido, T., et al.: 'ALIS evaluation tests in Croatia' SPIE - Defense, Security, and Sensing, 2009, pp. 73031B-73031B

[11] Daniels, D., Braunstein, J., Nevard, M.: 'Using minehound in Cambodia and Afghanistan', J. Conv. Weapons Destr., 2015, 18, (2), p. 14

[12] Geneva International Centre for Humanitarian Demining: 'MINEHOUND Trials 2005-2006: Sumamry Report'. Available at http://www.gichd.org/ resources/publications/

[13] Geneva International Centre for Humanitarian Demining: 'HOPE - Final Report'. Available at http://www.gichd.org/resources/publications/

[14] Zanzi, L., Lualdi, M., Braun, H.M., et al:: 'Ultrahigh-frequency radar sensor for humanitarian demining tested on different scenarios in 3D imaging mode'. 9th Int. Conf. on Ground Penetrating Radar (GPR2002), 2002, pp. 240-245

[15] Walls, R., Brown, T., Clodfelter, F., et al.: 'Ground penetrating radar field evaluation in Angola'. SPIE - Defense and Security Symp., 2006, pp. $62171 \mathrm{Y}-62171 \mathrm{Y}$

[16] Lombardi, F., Griffiths, H.D., Lualdi, M.: 'The influence of spatial sampling in GPR surveys for the detection of landmines and IEDs'. 2016 European Radar Conf, London, 2016, pp. 1-4

[17] Lualdi, M.: 'True' 3D acquisition using GPR over small areas: a cost effective solution'. 24th EEGS Symp. on the Application of Geophysics to Engineering and Environmental Problems, Chareston, April 2011, pp. 541-550

[18] Paglieroni, D.W., Chambers, D.H., Mast, J.E et al: 'Imaging modes for ground penetrating radar and their relation to detection performance', IEEE $J$ Sel. Top. Appl. Earth Obs. Remote Sens., 2015, 8, (3), pp. 1132-1144

[19] Groenenboom, J., Yarovoy, A.: 'Data processing and imaging in GPR system dedicated for landmine detection', Subsurf. Sens. Tech. Appl., 2002, 3, (4), pp. $387-402$

[20] Krueger, K.R., McClellan, J.H., Scott, W.R.: 'Efficient algorithm design for GPR imaging of landmines', IEEE Trans. Geosci. Remote Sens., 2015, 53, (7), pp. 4010-402

[21] Gonzalez-Huici, M.A., Giovanneschi, F. 'A combined strategy for landmine detection and identification using synthetic GPR responses', J. Appl. Geophys., 2013, 99, pp. 154-165

[22] Metwaly, M., Ismail, A., Matsushima, J.: 'Evaluating some factors that affect feasibility of using ground penetrating radar for landmine detection', Appl. Geophys., 2007, 4, (3), pp. 221-230

[23] Keeley, R.: 'Understanding landmines and mine action'. Available at http:// mit.edu/demining/assignments/understanding-landmines.pdf

[24] Lombardi, F., Griffiths, H.D., Balleri, A.: 'Influence of internal structure on landmine radar signatures'. 2016 European Radar Conf., London, 2016, pp. $1-4$
[25] Roberts, R.L., Daniels, J.J.: 'Analysis of GPR polarization phenomena', $J$ Environ. Eng. Geophys., 1996, 1, (2), pp. 139-157

[26] Watson, F., Lionheart, W.R.B.: 'SVD analysis of GPR full-wave inversion'. IEEE 15th Int. Conf. on GPR, Brussels, Belgium, June 2014, pp. 484-490

[27] Villela, A., Romo, J.M.: 'Invariant properties and rotation transformations of the GPR scattering matrix', J. Appl. Geophys., 2013, 90, pp. 71-81

[28] Lualdi, M., Lombardi, F.: 'Combining orthogonal polarization for elongated target detection with GPR', J. Geophys. Eng., 2014, 11, (5), p. 055006

[29] Lualdi, M., Lombardi, F.: 'Orthogonal polarization approach for three dimensional georadar surveys', NDT\&E Int., 2013, 60, pp. 87-99

[30] Boniger, U., Tronicke, J.: 'Subsurface utility extraction and characterization: combining GPR symmetry and polarization attributes', IEEE Trans. Geosci. Remote Sens., 2012, 50, (3), pp. 736-746

[31] Lualdi, M., Lombardi, F.: 'Utilities detection through the sum of orthogonal polarization in 3D georadar surveys', Near Surf. Geophys., 2015, 13, (1), pp 73-81

[32] Radzevicius, S.J., Guy, E.D., Daniels, J.J.: 'Pitfalls in GPR data interpretation: differentiating stratigraphy and buried objects from periodic antenna and target effects', Geophys. Res. Lett., 2000, 27, (20), pp. 3393 3396

[33] Lualdi, M., Lombardi, F.: 'Significance of GPR polarisation for improving target detection and characterisation', Nondestruct. Test. Eval., 2014, 29, (4) pp. $345-356$

[34] Streich, R., Van der Kruk, J.: 'Accurate imaging of multicomponent GPR data based on exact radiation patterns', IEEE Trans. Geosci. Remote Sens., 2007, 45, (1), pp. 93-103

[35] Radzevicius, S.J., Daniels, J.J. 'Ground penetrating radar polarization and scattering from cylinders', J. Appl. Geophys., 2000, 45, (2), pp. 111-125

[36] Núñez-Nieto, X., Solla, M., Gómez-Pérez, P., et al.: 'GPR signa characterization for automated landmine and UXO detection based on machine learning techniques', Remote Sens.., 2014, 6, (10), pp. 9729-9748

[37] Alawneh, I., Beine, C., Edenhofer, P.: 'Calculation of fingerprints of typical antipersonnel landmines by varying the observation point and incidence angles of excitations'. IEEE 6th European Conf. on Antennas and Propagation, Prague, March 2012, pp. 1068-1071

[38] Chun, E.H.Y., Chun, C.S.: 'Polarimetric invariants for detection by forwardlooking ground penetrating radar'. IEEE Radar Conf., Kansas City, May 2011, pp. 185-188

[39] Torrione, P.A., Morton, K.D., Sakaguchi, R., et al.: 'Histograms of oriented gradients for landmine detection in ground-penetrating radar data', IEEE Trans. Geosci. Remote Sens., 2014, 52, (3), pp. 1539-1550

[40] Zhu, Q., Collins, L.M.: 'Application of feature extraction methods for Zhu, Q., Collins, L.M.: 'Application of feature extraction methods for
landmine detection using the Wichmann/Niitek ground-penetrating radar', IEEE Trans. Geosci. Remote Sens., 2005, 43, (1), pp. 81-85

41] Langman, A., Inggs, M.R. 'Polarimetric model for a steppedfrequency continuous-wave ground penetrating radar', Proc. SPIE, 2002, 4758, pp. 645 651

[42] Wright, L., Balleri, A., Griffiths, H., et al.: 'Multi-perspective high range resolution profiles of landmines'. IEEE Radar Conf., 2015, Johannesburg, October 2015, pp. 51-55

[43] Marsland, T.P., Evans, S.: 'Dielectric measurements with an open-ended coaxial probe', IEE Proc. H, Microw. Antennas Propag., 2014, 134, (4), pp. 341-349

[44] Daniels, D.J., Curtis, P., Amin, R., et al: 'MINEHOUND production development'. SPIE - Defense and Security, 2005, pp. 488-494

[45] Sato, M., Yokota, Y., Takahashi, K.: 'ALIS: GPR system for humanitarian demining and its deployment in Cambodia', J. Electron. Eng. Sci., 2012, 12 (1), pp. 55-62

[46] Doheny, R.C., Burke, S., Cresci, R, et al. 'Handheld standoff mine detection system (HSTAMIDS) field evaluation in Thailand'. SPIE Defense and Security, 2005, pp. 889-900

[47] Soumekh, M., Ton, T., Howard, P.: 'NIITEK GPR', Proc. SPIE, 2009, 7308 p. $73080 \mathrm{~J}-1$

[48] Lualdi, M., Lombardi, F.: 'Effects of antenna orientation on 3-D ground penetrating radar surveys: an archaeological perspective', Geophys. J. Int., 2014, 196, (2), pp. 818-827

[49] Yilmaz, Ö.: 'Seismic data analysis' (Society of Exploration Geophysicists, 2001) 